\title{
The combination of exercise training and $\alpha$-lipoic acid treatment has therapeutic effects on the pathogenic phenotypes of Alzheimer's disease in NSE/APPsw-transgenic mice
}

\author{
JOON Y. $\mathrm{CHO}^{1}$, HYUN S. UM ${ }^{1}$, EUN B. KANG ${ }^{1}$, IN H. CHO ${ }^{1}$, \\ CHUL H. KIM ${ }^{1}$, JUNG S. CHO ${ }^{2}$ and DAE Y. HWANG ${ }^{3}$
}

\begin{abstract}
${ }^{1}$ Exercise Biochemistry Laboratory, Korea National Sport University, Seoul 138-763; ${ }^{2}$ Multidisciplinary Technology Institute, Hoseo University, Asan 336-795; ${ }^{3}$ Department of Biomaterial Science, College of Natural Resources and Life Science, Pusan National University/PNU-Laboratory Animal Resources Center, Miryang 627-706, Korea
\end{abstract}

Received October 13, 2009; Accepted December 16, 2009

DOI: $10.3892 /$ ijmm_00000350

\begin{abstract}
Exercise training was suggested as a practical therapeutic strategy for human subjects suffering from Alzheimer's disease (AD) in our previous study. Therefore, the purpose of this study was to investigate the effects of combining exercise training with the administration of antioxidants on the pathological phenotype of AD. To accomplish this, non-transgenic mice (Non-Tg) and NSE/ APPsw Tg mice were treated with $\alpha$-lipoic acid and treadmill exercised for 16 weeks, after which their brains were evaluated to determine whether any changes in the pathological phenotype-related factors occurred. The results indicated that (i) the combination-applied (COMA) Tg group with exercise training (ET) and $\alpha$-lipoic acid administration (LA) showed ameliorated spatial learning and memory compared to the sedentary (SED)-Tg and single-treatment groups; (ii) there were no differences in the level of $A \beta-42$ peptides across groups; (iii) the level of glucose transporter-1 and brain-derived neurotrophic factor proteins were highly increased in the COMA group, (iv) ET and LA did not induce a synergistic effect on the expression of heat shock protein-70 and apoptotic proteins including Bax and caspase-3; (v) the levels of SOD-1 and CAT suppressing oxidative stress
\end{abstract}

Correspondence to: Professor Joon Yong Cho, Exercise Biochemistry Laboratory, Korea National Sport University, 88-15 Oryun-dong, Songpa-gu, Seoul 138-763, Korea

E-mail: chojy86@knsu.ac.kr

Professor Dae Youn Hwang, Department of Biomaterial Science, Collage of Natural Resources and Life Science, Pusan National University, 50 Cheonghak-ri, Samnangjin-eup Miryang-si, Gyeongsangnam-do 627-706, Korea

E-mail: dyhwang@pusan.ac.kr

Key words: Alzheimer's disease, exercise training, $\alpha$-lipoic acid, brain-derived neurotrophic factor, glucose transporter-1 were extensively higher in the COMA than in the singletreated groups and (vi) there were no significant differences across groups regarding these serum characteristics, although these levels were lower than the SED-Tg group. Taken together, these results suggest that the combination with ET and LA may contribute to protect the neuron injury induced by $A ß$ peptides and may be considered an effective therapeutic strategy for human subjects suffering from AD.

\section{Introduction}

Alzheimer's disease (AD) is the most common form of dementia characterized by the progressive deterioration of cognitive functions with loss of memory concurrent with pathogenic alterations. Such pathogenic alterations are regarded as clues to the cause of $\mathrm{AD}$, particularly changes in neuronal cell metabolism such as the oxidation of proteins and DNA, lipid peroxidation, the formation of free radicals, the progression of neuritic injury by $\beta$-amyloid $(A B)$, the disruption of neuronal metabolic homeostasis and neuronal cell death (1-3). The neurotoxicity of $A \beta$, a major component of $\mathrm{AD}$ pathogenesis, has been suggested to induce neuronal degeneration and death by rendering neurons vulnerable to increases in oxidative stress and impairing cellular metabolism (4). In addition, AD has been found to be closely related to the mechanism of cell growth and death, involving the downregulation of growth factors (5) and up-regulation of caspasedependent neuronal apoptosis (6).

Furthermore, the phenotypical changes of AD are tightly linked to those of several chronic degenerative diseases, such as dysfunction of pancreatic $B$ cells associated with diabetes, which is also observed in AD patients. These phenotypes are also known to be involved in impaired cellular glucose transport activity (7), deranged expression of heat-shock proteins (8), hypercholesterolemia and hyperinsulinemia $(9,10)$. Taken together, the results of these studies suggest that therapeutic strategies for human chronic degenerative disease can be simultaneously applied against pathogenic phenotypes of $\mathrm{AD}$. Patients with $\mathrm{AD}$ have also been shown to exhibit weight loss, general weakness and fatigue, and 
reduced food intake coupled with decreased physical activity $(11,12)$.

In contrast, several epidemiological studies have shown that the transition from a sedentary to a simple active lifestyle may be sufficient to enhance cognitive ability and delay the onset of dementia in both humans and mice $(13,14)$. Therefore, exercise training (ET) has recently been included in AD treatment plans. Previous studies have suggested that ET could be beneficial to AD sufferers through multiple pathways by inducing improved spatial learning and memory, increased synaptic plasticity, cerebrovascular function and production of growth factors, as well as by reducing the formation of $\mathrm{A} \beta-42(15,16)$.

There is also increasing evidence that the neurodegenerative processes involved in $\mathrm{AD}$ may be associated with increased oxidative stress and that antioxidant therapy may be beneficial. $\alpha$-lipoic acid (LA) is a water soluble biological antioxidant (17) that can increase glucose metabolism by enhancing glucose transport and the activity of pyruvate dehydrogenase (18), as well as improve cognitive function and reduce brain oxidative stress $(19,20)$ and $A B$ induced neurotoxicity in hippocampal neurons (21). Notably, the importance of LA in the treatment of dementia was proposed as early as 1998 (22).

Although it is clear that treatment with ET or LA individually and in combination partially ameliorates pathogenic phenotypes of the brain in $\mathrm{AD}$, there is currently no information regarding how the combination of these two interventions might interact to ameliorate pathogenic phenotypes in AD animal models that express human-like phenotypes. Therefore, this study was conducted to determine whether the combination of ET and chronic treatment with the $\mathrm{R}(+)$-enantiomer of lipoic acid improves the molecular pathogenic phenotypes of $\mathrm{AD}$ to a greater extent than either treatment administered individually, using a novel AD model that expresses the human APPsw gene. Our results demonstrated that the combination of ET and LA had a synergic effect on the behavioral impairment and expression of several key proteins including brain-derived neurotrophic factor (BDNF), glucose transporter-1 (GLUT-1), superoxide dismutase-1 (SOD) and catalase (CAT) in NSE/APPsw Tg mice.

\section{Materials and methods}

Transgenic mice. All animal experimental procedures used in this study were approved by the Institutional Animal Care and Use Committee (IACUC) at the Korea National Sport University and the Korea FDA. NSE/APPsw Tg mice expressing the human APPsw gene under the control of the neuron-specific enolase (NSE) promoter were used for this study. These mice present AD phenotypes, including behavioral dysfunction, Aß-42 deposition and apoptosis activation at 12-13 months of age (23). The Non-Tg and Tg mice were obtained from the Department of Laboratory Animal Resources at the National Institute of Toxicological Research, Korea FDA. The mice were handled in an animal facility accredited by the Korea FDA according to the AAALAC International Animal Care Policies (Accredited Unit-Korea Food and Drug Administration: unit no. 000996).
Mice were maintained under a 12:12-h dark-light cycle at $22 \pm 2{ }^{\circ} \mathrm{C}$ and $50 \%$ relative humidity and were provided with a standard chow diet (Purina Mills Inc.) ad libitum.

Experimental design and treatment. Thirteen-month-old NSE/APPsw Tg mice $(n=20)$ and Non-Tg mice $(n=5)$ were assigned to one of the following groups: sedentary Non-Tg mice (SED-Non-Tg), sedentary Tg mice (SED-Tg), exercisetrained Tg mice (ET-Tg), $\alpha$-lipoic acid-administered Tg mice (LA-Tg), or Tg mice applied the combination with ET and LA (COMA-Tg). Animals in the LA-Tg group received $100 \mathrm{mg} / \mathrm{kg}$ body weight/day of purified $\alpha$-lipoic acid (T5625, DL-6, 8-thioctic acid, Sigma-Aldrich, St. Louis, MO, USA), dissolved in $1 \mathrm{~N} \mathrm{NaOH}$ and brought to neutrality with $1 \mathrm{~N}$ $\mathrm{HCl}$ by intraperitoneal injection for 16 weeks. The SED-Non$\mathrm{Tg}$ and $-\mathrm{Tg}$ mice received $8.3 \mathrm{ml} / \mathrm{kg}$ body weight/day of vehicle, except for $\alpha$-lipoic acid as previously described (24). Mice in the ET-Non-Tg and -Tg groups ran for $60 \mathrm{~min}$ a day, $13.2 \mathrm{~m} / \mathrm{min}$ for 5 days/week on a $0 \%$ gradient for 16 weeks. Prior to the treadmill exercise training, the mice were allowed a minimum of 1 week of familiarization with the new environment, which included running for $10 \mathrm{~min}$ a day on the treadmill (11 m/min), as previously described (13). The animals in the COMA-Tg group also performed the treadmill training protocol exactly as described above while also receiving a daily treatment with $\alpha$-lipoic acid.

Water maze test. The mice were subjected to water maze tests following previously described procedures (25). Briefly, the tests were performed using the Smart-CS program (Panlab, Barcelona, Spain), which was placed in an experimental room with a window, air-conditioning and tables. This experiment was conducted in a plastic circular pool with a diameter of $1.5 \mathrm{~m}$ that was filled with water maintained at $20-22^{\circ} \mathrm{C}$. The visual field of the water was obstructed by the addition of powered milk. Mice were pre-trained by allowing them to swim to a round platform (diameter $12 \mathrm{~cm}$ ) submerged $1 \mathrm{~cm}$ beneath the surface. The escape latencies, escape distances, swimming speeds and swimming patterns of the mice were monitored by the Smart-LD computer program, which was connected to a camera mounted to the ceiling directly above the pool. Prior to the experiment, a 60-sec habituation trial was performed to verify that the mice could swim. The mice were then given five training trials in which their ability to find the hidden platform was measured for a maximum of $60 \mathrm{sec}$. If the mice failed to find the platform within the maximum time, they were physically placed on it. The training schedule consisted of two trials per day over 5 test days, and each trial was assessed based on the ability of the mouse to reach the platform within $60 \mathrm{sec}$. The second trial was conducted at least $5 \mathrm{~min}$ after the first one. However, the two trials were started from identical locations, and the platform location was kept constant during the training period. After each trial, the mice were allowed to remain on the platform for $30 \mathrm{sec}$. On the sixth day, the mice were subjected to three probe trials, during which they swam for $60 \mathrm{sec}$ with no platform in the pool. In this test, each of the two training and three actual trials were initially started from the right side of the water pool, and then from the opposite side. The patterns of searching, the number of times the mice 
swam to the former location of the platform (escape latency), the distances the mice swam (escape distance) and the swimming speeds (velocity) to the former location of the platform were recorded. All trials were recorded and stored on video-tape for subsequent analysis.

Western blot analysis. Western blot analyses were conducted as previously described (23). Briefly, proteins (40 $\mu \mathrm{g})$ were separated by electrophoresis on a $10 \%$ polyacrylamide gel for $3 \mathrm{~h}$, after which they were transferred to a nitrocellulose membrane (Immuno-Blot, PVDF membrane, Bio-Rad, CA, USA) for $2 \mathrm{~h}$ at a constant voltage of 40 volts. Each membrane was then separately incubated overnight at $4^{\circ} \mathrm{C}$ with the primary antibodies: anti-Aß-42 (MAB1561, Chemicon, MS, USA), anti-SOD1 (sc-11407, Santa Cruz Biotechnology, CA, USA), anti-HSP70 (sc-24, Santa Cruz Biotechnology), anticatalase (sc-33781, Santa Cruz Biotechnology), anti-BDNF (sc-546, Santa Cruz Biotechnology), anti-GLUT-1 (sc-7903, Santa Cruz Biotechnology), anti-Bcl-2 (C9804, Sigma, MI, USA), anti-Bax (B8429, Sigma), anti-caspase-3 (C8487, Sigma), and anti-GADPH (sc-20357, Santa Cruz Biotechnology). The membranes were then washed with washing buffer, after which they were incubated with secondary antibodies, horseradish peroxidase-conjugated goat anti-rabbit (65-6120, Zymed Lab, CA, USA) for BDNF, SOD-1 and caspase-3 (1:3000); horseradish peroxidaseconjugated goat anti-mouse (sc-2005, Santa Cruz Biotechnology) for B-amyloid, Bax, Bcl-2, and HSP70 (1:3000); and horseradish peroxidase-conjugated goat anti-goat (81-1620, Zymed Lab) for SOD-1 (1:3000). The membrane blots were then developed using a Western blot analysis system (Santa Cruz Biotechnology) following the manufacturer's instructions. The density of the developed bands was determined using a ChemiDoc XRS System (Bio-Rad, Hercules, CA, USA).

Biochemical analysis. Following the final application of ET and/or LA, the mice were fasted for $24 \mathrm{~h}$, after which whole blood was collected from their abdominal vein. Serum was obtained by centrifuging the blood $\left(15,000 \mathrm{rpm}, 4^{\circ} \mathrm{C}, 10 \mathrm{~min}\right)$ followed by incubation for $30 \mathrm{~min}$ at room temperature. The serum was then stored at $-80^{\circ} \mathrm{C}$ until analysis. The total serum cholesterol (Cholesterol Reagent Kit) and glucose (Glucose Hexokinase Kit) (both kits from Bayer, Pittsburgh, PA, USA) were assayed using the appropriate kits and following standard methods.

ELISA for insulin detection. The levels of serum insulin obtained from Non-Tg and Tg mice were detected using the ultra-sensitive assay procedure and reagents in the insulin ELISA kit (cat. 10-1137-01, Mercodia, Sweden). Briefly, sera and standards were incubated in a plate shaker at 100$150 \mathrm{rpm}$ at room temperature for $2 \mathrm{~h}$ on antibody-coated plates. The wells were then washed six times with an automatic plate washer (PV100, Hoefer, USA), after which HRP conjugate was added to each of the wells. The plates were then incubated in a shaker for $30 \mathrm{~min}$ at room temperature. The reaction was terminated by the addition of $50 \mu 1$ of stop solution $\left(0.5 \mathrm{M} \mathrm{H}_{2} \mathrm{SO}_{4}\right)$, after which the plates were analyzed by evaluating the absorbance at $450 \mathrm{~nm}$ using a
Molecular Devices $\mathrm{V}_{\max }$ Plate Reader (Molecular Devices, Sunnyvale, CA, USA).

Statistical analysis. Data were analyzed using SPSS (version 10.0, SPSS Inc., Chicago, IL, USA). All values were expressed as means \pm SE. When comparing groups, statistical significance was determined by one-way ANOVA. If a statistically significant difference was observed, the Student Newman-Keuls post hoc test was used. The differences were considered to be statistically significant at $\mathrm{p}<0.05$.

\section{Results}

Preventative effects of exercise training and a-lipoic acid treatment on spatial learning and memory deficits. Previous studies have demonstrated that the APPsw Tg mice used in this study exhibit behavioral deficits at 13 months of age, and that these deficits are accompanied by increased levels of AB-42 (23). Therefore, the initial purpose of the study was to estimate whether treatment with ET alone, LA alone or COMA affected the behavioral dysfunction of AD pheno-types. Using water maze tests, the escape distances, swimming speed, escape latencies and swimming patterns were estimated for each group. In the SED groups, Tg mice swam for a longer distance, had a higher latency and slower velocity than mice in the Non-Tg group $(\mathrm{p}<0.05)$ (Fig. 1). However, ET alone, LA alone or COMA resulted in a significantly shorter distance and lower latency, as well as a significantly higher velocity than was observed in the SED-Tg mice (all p<0.05) (Fig. 1). The COMA group showed a higher efficiency than either single-treated group in the improvement of behavioral dysfunction. Taken together, these observations suggested that COMA effectively contributed to the improvement in the spatial learning and memory deficits of $\mathrm{AD}$ in the brains of $\mathrm{Tg}$ mice compared to the single-treated groups.

Preventative effects of exercise training and $\alpha$-lipoic acid treatment on Aß-42 deposition in the brains of NSE/APPsw $T g$ mice. To investigate the preventative effects of ET and LA on Aß-42 deposition, Non-Tg and Tg mice that were 13 months of age were subjected to one of the aforementioned treatments for 16 weeks. The Aß-42 levels were subsequently measured in the mouse brain extracts of the five groups by Western blot analysis. SED-Tg mice had a higher level of $\mathrm{A} \beta-42$ than SED-Non-Tg mice $(\mathrm{p}<0.05)$. However, Aß-42 protein levels in the brains of ET alone, LA alone or COMA mice were much lower than those of SED-Tg mice, although the rate at which these levels decreased differed among the groups (all $\mathrm{p}<0.05)$ (Fig. 2). Taken together, these results suggest that COMA leads to a reduction in the levels of Aß-42 in the brain, although the level of this reduction was very similar to the ET alone or LA alone groups.

Neuroprotective effects of exercise training and $\alpha$-lipoic acid treatment on the BDNF and GLUT-1 protein levels in the brains of NSE/APPSW Tg mice. BDNF has been shown to exert beneficial effects on synaptic plasticity and may facilitate learning and memory. It has been reported that $\mathrm{AD}$ may be caused or exacerbated by low levels of BDNF in the basal 
A
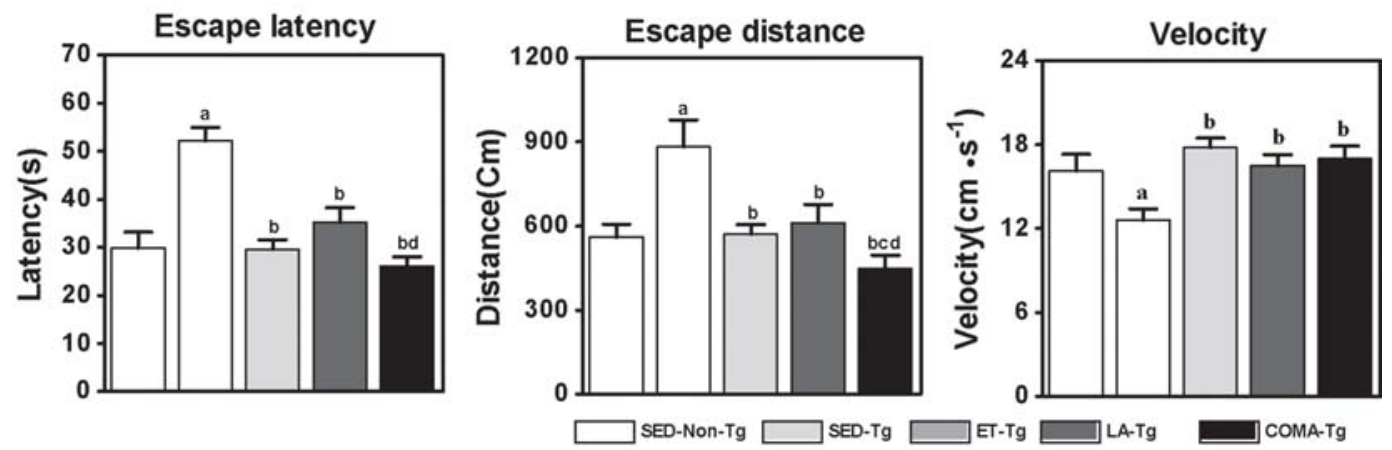

B

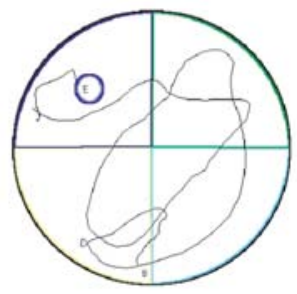

SED-Non-Tg

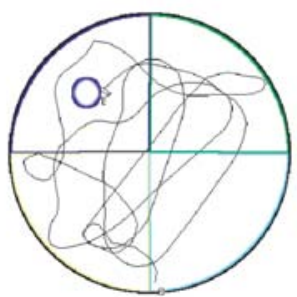

SED-Tg

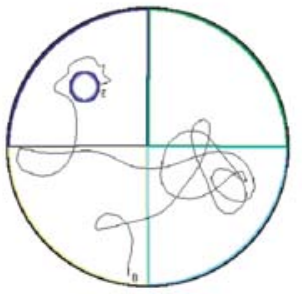

ET-Tg

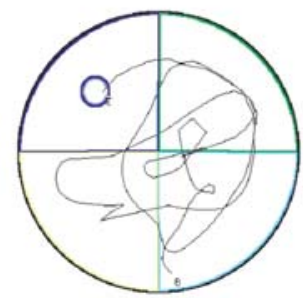

LA-Tg

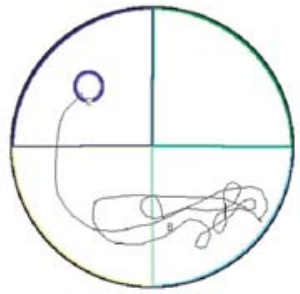

COMA-Tg

Figure 1. Data (A) and patterns (B) of escape latency, escape distance and swimming velocity of mice searching for the former platform location in the water maze. APPsw Tg mice showed a significantly different trend toward escape latency, distance and velocity upon exercise training. Swimming patterns showed the difference in mice activity in the ET, LA, and COMA groups when crossing the former platform location. Five mice per each group were assayed in triplicate. Values are shown as the mean \pm SE. ${ }^{a} \mathrm{p}<0.05$ vs the SED-Non-Tg group. ${ }^{b} \mathrm{p}<0.01$ vs the SED-Tg group. ${ }^{\mathrm{c}} \mathrm{p}<0.001$ vs the ET-Tg group. ${ }^{\mathrm{d}} \mathrm{p}<0.001 \mathrm{vs}$ the LA-Tg group.

A

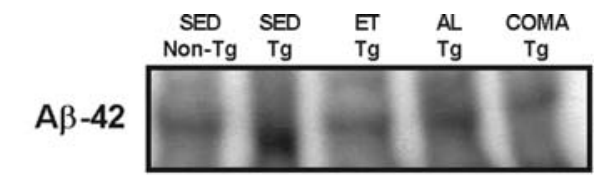

B

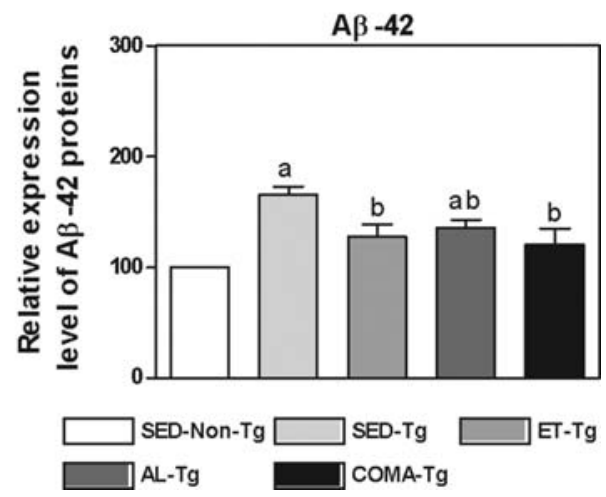

Figure 2. Aß-42 deposition in the brains of APPsw Tg mice subjected to ET, LA, or COMA for 16 weeks. The levels of AB-42 in the brain were analyzed by Western blot analysis with the human Aß-42-specific antibody. Five mice per each group were assayed in triplicate. Values are shown as the mean \pm SE. ${ }^{a} p<0.05$ vs the SED-Non-Tg group. ${ }^{b} p<0.01$ vs the SED-Tg group. forebrain (5). Therefore, to determine whether ET alone, LA alone or COMA had any effect on synaptic plasticity, spatial learning and memory in the brains of APPsw $\mathrm{Tg}$ mice, the expression level of BDNF was estimated by Western blot analysis. SED-Tg mice had a lower level of BDNF than SED-Non-Tg mice ( $\mathrm{p}<0.05$ ) (Fig. 3), and BDNF protein levels in the brains of ET alone and LA or COMA groups were significantly greater compared to those of the SED-Tg group. Of these groups, COMA had significantly higher levels than either single-treatment group (all p<0.05) (Fig. 3).

In addition, although GLUT-1 is selectively expressed at high levels in the capillary endothelium of the brain and is responsible for the transfer of glucose across the blood-brain barrier (26), the expression of GLUT-1 is decreased in the hippocampus and cerebral cortex of AD patients (27). These studies indicate that the reduction of GLUT-1 reflects the diminution of glucose metabolism and possibly a hypometabolic state. Therefore, GLUT-1 protein levels were determined to ascertain whether they respond to ET alone, LA alone or COMA in a similar fashion to AD patients. Western blot analysis revealed that the GLUT-1 levels in the brains of mice subjected to ET alone, LA alone or COMA 


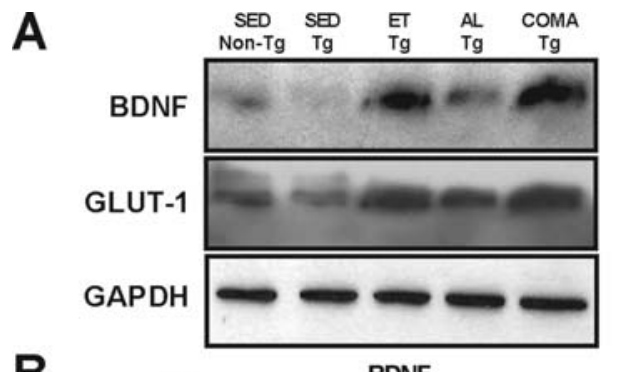

B
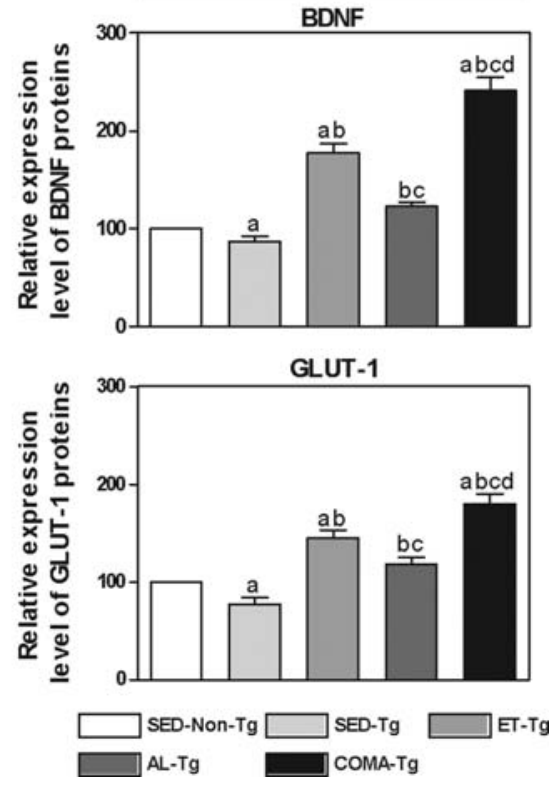

Figure 3. Expression of BDNF and GLUT-1 proteins in the brains of APPsw $\mathrm{Tg}$ mice subjected to ET, LA, or COMA for 16 weeks. The expression levels of BNDF and GLUT-1 proteins in the brain were analyzed by Western blot analysis. Five mice per each group were assayed in triplicate. Values represent the mean \pm SE. a $\mathrm{p}<0.03$ vs the SED-Non-Tg group. ${ }^{b} \mathrm{p}<0.04$ vs the SED-Tg group. ${ }^{\mathrm{p}}<0.002$ vs the ET-Tg group. ${ }^{\mathrm{d}} \mathrm{p}<0.003$ vs the LA-Tg group.

were higher than in the SED-Tg group, although the highest rate at which these levels decreased was observed in the COMA group (all p<0.05) (Fig. 3B). In addition, SED-Tg mice had a lower level of GLUT-1 than SED-Non-Tg mice $(\mathrm{p}<0.05)$ (Fig. 3). These results indicate that COMA for 16 weeks may be directly involved with synaptic plasticity, neuroprotection and glucose metabolism by regulating the expression level of BDNF and GLUT-1 proteins.

Anti-apoptotic effects of exercise training and a-lipoic acid treatment on the apoptosis pathway in the brains of NSE/ APPsw Tg mice. Mutations in the gene encoding for (apoptosis-related proteins) APP have been shown to cause $\mathrm{AD}$ and to lead to the production of $\mathrm{A} B$. As $\mathrm{A} \beta$ production may drive cells into apoptosis and activate the cell death signal pathway, Aß-induced neuronal death is considered to be caused by the caspase- 3 and Bcl- 2 families. Among the caspase families, caspase- 3 is involved in APP processing, which is consistent with the elevation of $A B$ formation that is observed in the neurons of AD patients (28). ET alone, LA alone or COMA were evaluated to determine whether they were able to prevent or ameliorate cell death in the brains of 13-month-old Tg mice, as in AD patients. Western blot analysis showed that the brains of SED-Tg mice had a higher level of Bax and caspase-3 than those of SED-Non-Tg mice

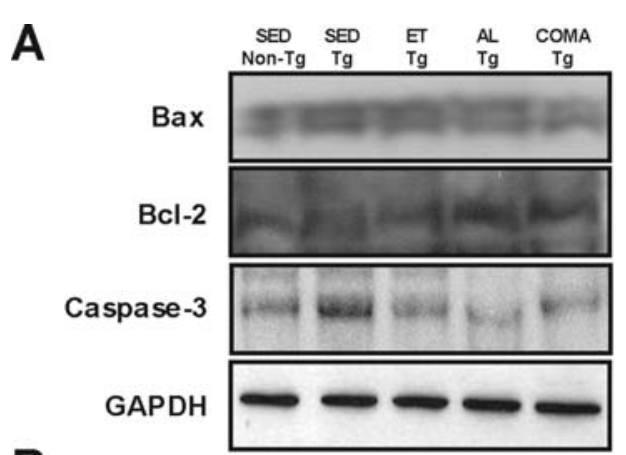

B
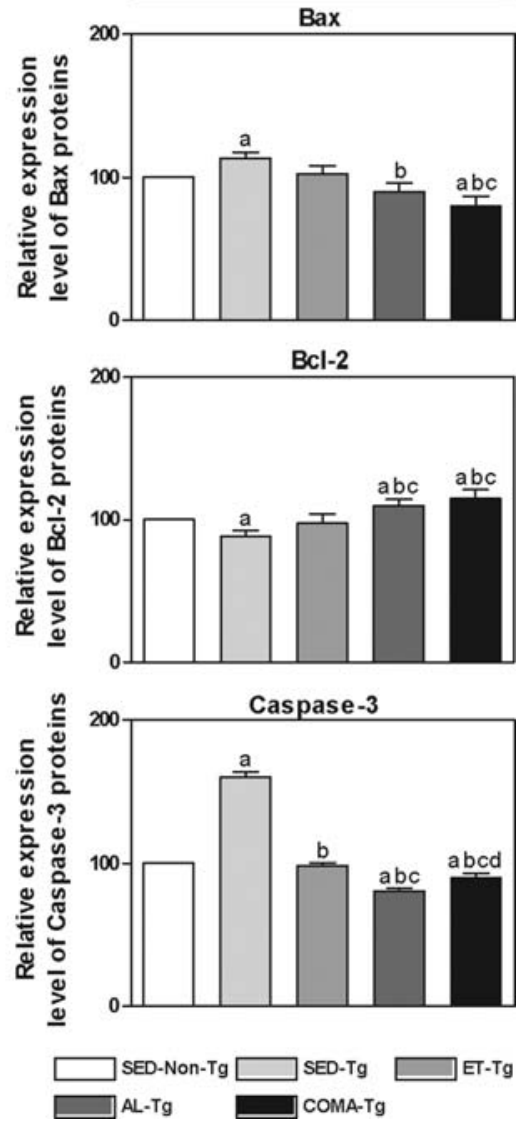

Figure 4. Expression of Bax, Bcl-2 and caspase- 3 proteins in the brains of APPsw Tg mice subjected to ET, LA, or COMA for 16 weeks. The expression levels of three proteins in the brain were detected by Western blot analysis. Five mice per each group were assayed in triplicate. Values represent the mean \pm SE. ${ }^{\mathrm{a}} \mathrm{p}<0.04$ vs the SED-Non-Tg group. ${ }^{\mathrm{b}} \mathrm{p}<0.05$ vs the SED-Tg group. ${ }^{c} \mathrm{p}<0.004$ vs the ET-Tg group. ${ }^{\mathrm{d}} \mathrm{p}<0.002$ vs the LA-Tg group.

$(\mathrm{p}<0.05)$ (Fig. 4). However, the Bax and caspase-3 levels in the brains of mice subjected to ET alone, LA alone or COMA were much lower than levels in the SED-Tg group, although the rate at which these levels decreased differed among groups (all $\mathrm{p}<0.05)$. Also, the Tg mice subjected to LA alone or COMA were found to have a higher level of Bcl-2 than the SED-Tg mice (all $\mathrm{p}<0.05)$, but no difference was observed between ET-Tg and SED-Tg groups (Fig. 4). Furthermore, these protein levels in the COMA group did not show a significant difference compared to levels in the ET or LA alone groups. Therefore, these results suggest that the COMA did not have a synergic effect in attenuating apoptosis in neuronal cells during AD pathogenesis by regulating the expression levels of the apoptosis-related proteins. 
A
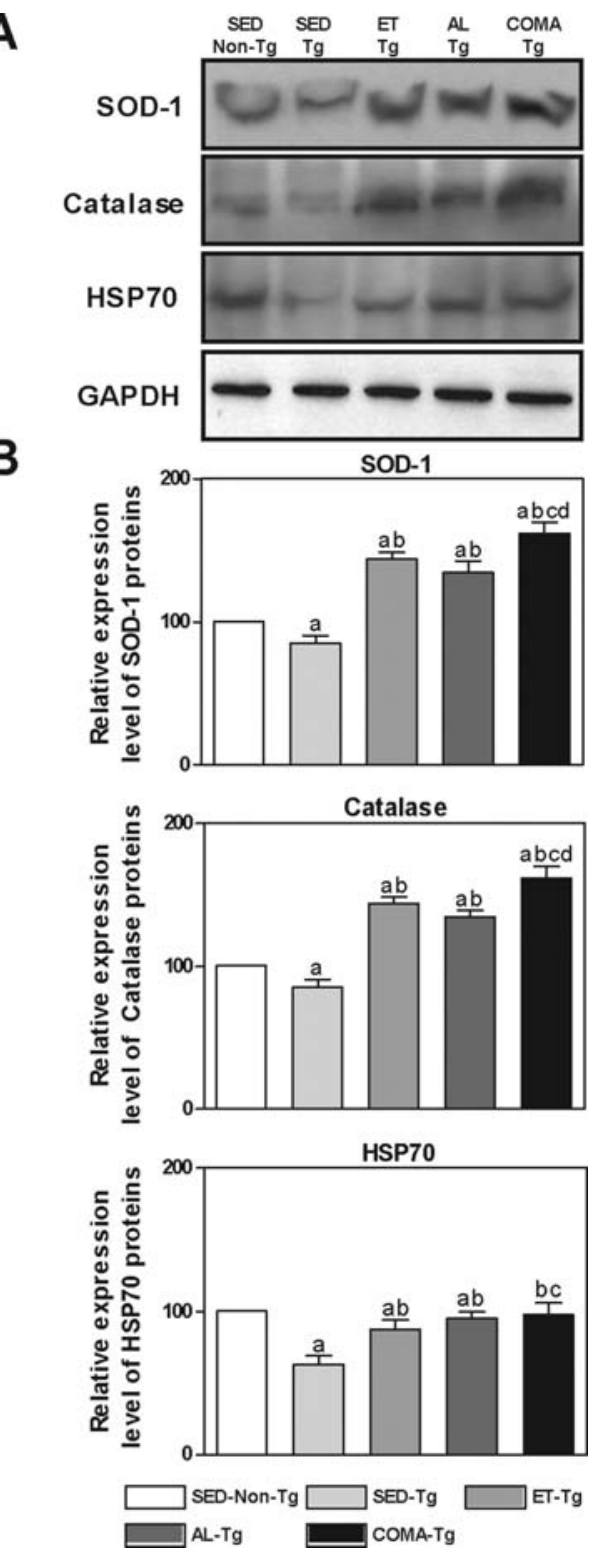

Figure 5. Expression of superoxide dismutase-1 (SOD-1), catalase (CAT) and heat shock protein-70 (HSP-70) in the brains of APPsw Tg mice subjected to ET, LA, or COMA for 16 weeks. The expression levels of the three proteins in the brain were detected by Western blot analysis. Five mice per each group were assayed in triplicate. Values represent the mean $\pm \mathrm{SE}$ ${ }^{\mathrm{a}} \mathrm{p}<0.01$ vs the SED-Non-Tg group. ${ }^{\mathrm{b}} \mathrm{p}<0.03$ vs the SED-Tg group. ${ }^{\mathrm{c}} \mathrm{p}<0.003$ vs the ET-Tg group. ${ }^{\mathrm{d}} \mathrm{p}<0.002$ vs the LA-Tg group.

Synergistic effects of exercise training and $\alpha$-lipoic acid treatment on SOD-1, CAT activity and HSP-70 expression in the brain of NSE/APPsw Tg mice. The SOD-1 and CAT activities of $\mathrm{AD}$ patients appear to be very low when compared with non-AD patients. Therefore, the effects of ET alone, LA alone or COMA were evaluated to determine whether SOD-1 and CAT expression increased in the brains of the Tg mice, as in AD patients. In the SED group, the brains of Tg mice were found to have slightly lower levels of SOD-1 and CAT proteins than those in Non-Tg mice $(\mathrm{p}<0.05)$ (Fig. 5). These protein levels were significantly increased (all $\mathrm{p}<0.05)$ with ET alone or LA alone (Fig. 5). COMA induced greater expression of SOD-1 than either single-treatment group. The
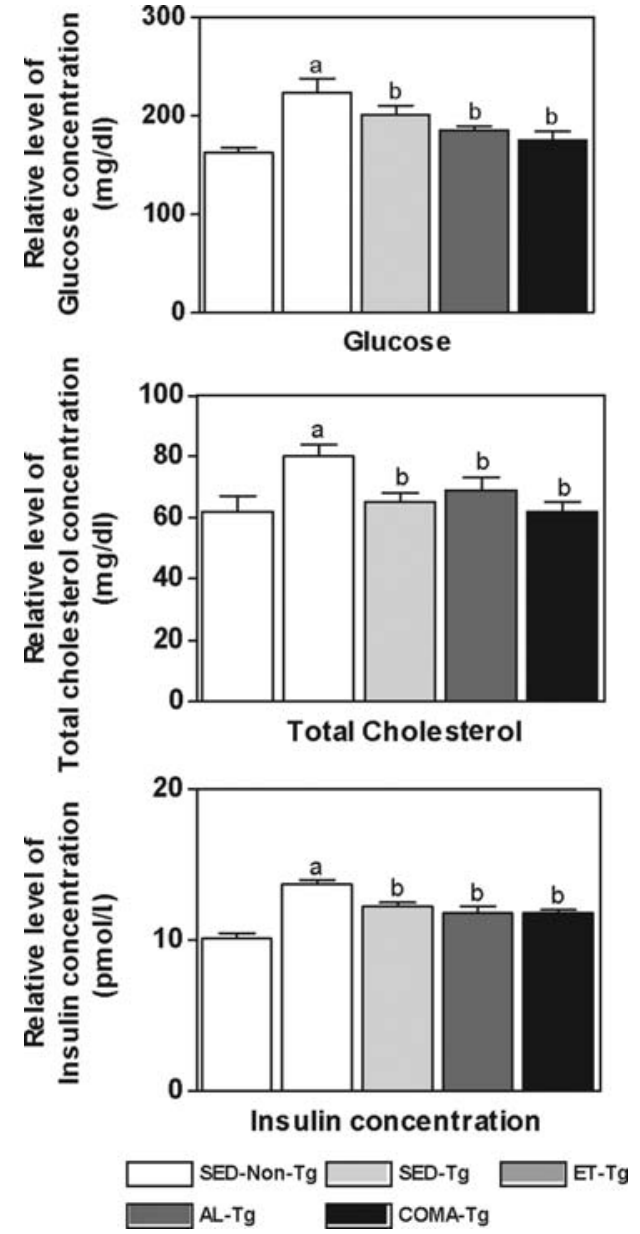

Figure 6. Serum biochemical profile of the APPsw transgenic mice subjected to ET, LA, or COMA for 16 weeks. Blood was collected from the abdominal vein of the mice, and total cholesterol (TC), glucose, and insulin concentrations in the serum were analyzed; five mice were assayed in triplicate. Values shown are the mean \pm SE. ${ }^{a} p<0.03$ vs the SED-Non-Tg group. ${ }^{b} \mathrm{p}<0.04$ vs the SED-Tg group.

findings obtained in this study indicate that COMA effectively induces a significant increase in the antioxidant capacity of the brains of $\mathrm{Tg}$ mice.

HSP-70 is a highly conserved protein that protects neuronal cells against excitotoxic and oxidative injury (30). This protection is partially related to the inhibition of apoptosis (31). Although the function of HSP-70 in AD neuropathology has been suggested, the expression pattern of this molecular chaperone in AD-affected brains is poorly understood. More importantly, the results of the present study provide an explanation for the mechanism by which ET alone, LA alone or COMA can affect cell protection in the brain cells of Tg mice. Therefore, we investigated HSP-70 protein levels to determine whether they were increased in the brains of $\mathrm{Tg}$ mice as in $\mathrm{AD}$ patients. In the SED group, the levels of HSP-70 were much lower in the Tg mice than in the Non-Tg mice $(\mathrm{p}<0.05)$ (Fig. 5). Following 16 weeks of ET alone, LA alone or COMA, the expression levels of HSP-70 protein were significantly higher in the $\mathrm{Tg}$ mice than in the SED-Tg mice (all p<0.05) (Fig. 5). COMA did not show a synergic effect on HSP70 expression. These results suggest that ET alone, LA alone or COMA had the same effects on the 
expression level of HSP70 to protect against the pathological changes of $\mathrm{AD}$.

Restoration effects of exercise training and $\alpha$-lipoic acid treatment on the change of metabolic parameters in the brains of NSE/APPSw Tg mice. The complex association between cholesterol, glucose, insulin, and AD has been investigated in previous studies (32). High levels of total cholesterol (TC) and insulin affect AB synthesis and APP metabolism since TC levels modulate $\gamma$-secretase activity and $A ß$ synthesis (9). Furthermore, there is growing evidence that impairments in insulin signaling are partly responsible for the cognitive decline that occurs in $\mathrm{AD}$, and it has been suggested that glucose metabolic abnormalities such as hyperglycemia and hyperinsulinemia are contributing or associated factors. Therefore, TC, glucose and insulin levels were assessed to determine whether ET alone, LA alone or COMA impacted these metabolic parameters. Prior to any treatment, the brains of Tg mice had a higher level of TC, glucose and insulin than those of Non-Tg mice $(\mathrm{p}<0.05)$ (Fig. 6). After 16 weeks of ET, LA, or COMA, all three factors were significantly lower in Tg mice than in SED-Tg mice (all $\mathrm{p}<0.05$ ). However, there were no significance differences between single-treatment groups and the COMA group. Taken together, these results suggest that ET alone, LA alone or COMA is a feasible and effective method for reducing TC, glucose and insulin levels in patients with $\mathrm{AD}$.

\section{Discussion}

The physical and mental benefits of physical activity and antioxidants are widely known, but seldom available to people suffering from AD. Although it may not be possible to prevent or change the course of the underlying disease, the benefits of ET and the antioxidant, $\alpha$-lipoic acid, in the management of AD patients have been demonstrated. ET is regarded as a useful tool to ameliorate cognitive and physical decline and maintain the quality of life of $\mathrm{AD}$ patients $(33,34)$. Moreover, $\alpha$-lipoic acid is a strong water-soluble antioxidant that also improves cognitive function and reduces brain oxidative stress $(19,20)$.

Since the early 1990 s, $\alpha$-lipoic acid has been used as a dietary supplement for humans, typically at doses in the range of 60-200 mg/day. In addition, the no observable adverse effect level (NOAEL) is considered to be $60 \mathrm{mg} / \mathrm{kg} \mathrm{wt} /$ day (35). However, despite growing evidence of the benefits of ET or LA for the health and function of AD patients, there is little clinical evidence that supports the recommendations for $\alpha$-lipoic acid supplementation and ET guidelines in AD patients. Moreover, there are many social and practical issues that prohibit recruiting individuals with $\mathrm{AD}$ for the purpose of scientific study. Therefore, we employed APPsw Tg mice, exhibiting learning and memory deficits in addition to elevated levels of $A ß-42$ in the brain to evaluate the protective effects of ET and LA individually or COMA on molecular pathogenic phenotypes associated with AD.

The results of the present study demonstrated that ET significantly improved spatial learning and memory deficits associated with APP overexpression in APPsw Tg mice, while reducing measurable brain levels of $\mathrm{A} ß$. These findings are in agreement with previous studies $(13,16)$ and suggest that ET can be used to prevent the accumulation of APP since it induces increased degradation and clearance of $A B$ $(16,36)$. Furthermore, $\alpha$-lipoic acid is a strong, water soluble antioxidant that also protects hippocampal neurons from $A ß-$ induced neurotoxicity (21), improves cognitive function and reduces brain oxidative stress $(19,20)$.

In this study, APPsw Tg mice were treated with $100 \mathrm{mg} /$ $\mathrm{kg} \mathrm{wt/day} \alpha$-lipoic acid, which is similar to the dose that effectively alleviates memory loss and oxidative stress in older rats (20). The $\alpha$-lipoic acid treatment reduced the cognitive deficits associated with APP overexpression in aged APPsw Tg mice while affecting measurable brain levels of $A \beta$, similar to the results by Lovell et al (21), who suggested that $\alpha$-lipoic acid had neuroprotective effects against Aß-mediated cytotoxicity. Notably, the results of this study demonstrated that application of a combination of ET and LA induced greater elevations in muscle-stimulated degradation and clearance of $A B$ or LA induced the radical scavenger properties on $\mathrm{A} \beta$-mediated cytotoxicity as well as improved the cognitive function. These findings indicate that ET reduced the amount of $A ß$ plaques in the brain, possibly via regulation of the APP process or increased degradation and clearance of $\mathrm{A} \beta$.

One important finding of this study was that ET alone or LA alone significantly up-regulated BDNF protein levels and improved cognitive deficits in APPsw Tg mice. Other studies have shown that ET alone or LA alone was sufficient to enhance spatial learning and memory and growth factors in transgenic mice and human AD patients (16,37). These findings were confirmed by the results of this study, which demonstrated that it was possible to elicit positive spatial learning and memory changes and increase BDNF protein levels in APPsw Tg mice with ET or LA alone.

In addition, the combination of ET and LA induced greater benefits in spatial learning and memory enhancement, as well as a further increase in BDNF protein levels than either treatment alone. These findings suggest that a combination of ET and LA could be more beneficial to the brain in terms of BDNF levels. Thus, these findings indicate that the combined protocol improves spatial learning and memory changes and increases BDNF protein levels via up-regulation of the cellular antioxidant system and reduction in the Aß-42 peptide load in the brain of APPsw Tg mice.

The expression of GLUT-1 protein has been found to be decreased in the brains (hippocampus, cerebral cortex) of AD $\mathrm{Tg}$ mice and humans $(7,27)$. These findings are in accordance with the results of this and previous studies $(38,39)$. Decreased GLUT-1 protein levels may result from a decreased GLUT-1 level/capillary density or may be caused by Aß-42 deposition (40). Conversely, ET may decrease brain cell damage caused by oxygen and glucose deprivation while increasing the level of insulin receptors that stimulate glucose uptake mediated by the activation of GLUT-1 (41). In addition, $\alpha$-lipoic acid has the ability to increase glucose utilization in vivo, which may be relevant to the treatment of neurodegenerative diseases (42). However, the exact mechanism by which ET alone or LA alone affects GLUT-1 protein levels in AD is still unknown. Furthermore, there are no reports evaluating the effects of a combination of ET and LA on GLUT-1 protein levels using 
AD patients or Tg mice. Therefore, ET alone, LA alone or COMA was evaluated to determine whether it increased GLUT-1 protein levels in the brains of 13-month-old APPsw Tg mice. The results revealed a dramatic increase in GLUT-1 protein in the brains of ET alone, LA alone or COMA mice when compared with the SED-Tg mice. The increased GLUT-1 content in Tg mice treated with ET or LA alone or with COMA may have occurred due to a clinical reduction in blood metabolic parameters (TC, glucose, insulin) and Aß-42 levels in response to these treatments, possibly through a systemically improved neuronal insulin signal transduction cascade that likely involves promotion of GLUT-1 synthesis or a reduction in GLUT-1 degradation $(21,43)$. These findings indicate that ET alone or LA alone exerts a stimulatory effect on the uptake and utilization of glucose in APPsw Tg mice. These effects were observed as higher levels in the COMA group.

Excessive or inappropriate apoptosis has been implicated in several neurodegenerative disorders including AD, Parkinson's disease (PD) and Huntington's disease (HD). Notably, AD shows high levels of Aß-42 deposition that promotes neuronal cell death mediated by apoptotic-related pathways or apoptosis itself. Conversely, ET reduces amyloid- $\beta$ plaques and improves cognitive function, synaptic plasticity, and neurogenesis in AD Tg mice $(13,44)$. Furthermore, LA ameliorates cognitive dysfunction and reduces $\mathrm{A} \beta$-induced oxidative damage in the brains of $\mathrm{AD} \mathrm{Tg}$ mice $(37,42)$. However, the exact mechanisms that regulate the effects of ET and LA either individually or in COMA on AD-related apoptosis are still unclear. Indeed, this is the first study to examine the effects of ET and LA alone and in combination on Bax, caspase- 3 and $\mathrm{Bcl}-2$ protein expression in the brains of APPsw Tg mice. The results of the present study revealed that Bax and caspase- 3 protein levels were significantly lower in the brains of $\mathrm{Tg}$ mice following treatment with ET alone, LA alone or COMA, whereas the $\mathrm{Bcl}-2$ protein level was significantly increased in the brains of mice subjected to ET alone, LA alone or COMA when compared with SED-Tg mice. These results indicate that the reduction of the pro-apoptotic Bax protein and the induction of anti-apoptotic $\mathrm{Bcl}-2$ protein via reduction of caspase- 3 following either ET or LA in APPsw Tg mice may be indirectly linked to a reduction in the $\mathrm{A} \beta-42$ protein level, possibly via up-regulation of antioxidative molecules (SOD, CAT) (16). These findings indicate that either ET or LA can alleviate the neuronal cell death associated with AD by increasing the degradation and clearance of $A B$ or antioxidative molecules which is consistent with the results of several previous studies $(21,36)$. Surprisingly, the addition of ET to LA-subjected-Tg mice elicited a greater up-regulation of $\mathrm{Bcl}-2$ and down-regulation of Bax and caspase-3 proteins than ET or LA alone. One possible explanation for these findings is that COMA induced a greater neuronal resistance against apoptotic and oxidative injury than ET or LA alone, possibly via an increase in Bcl-2 and a decrease in Bax, caspase- 3 protein and $A B$ deposition.

In neurodegenerative disorders such as $\mathrm{PD}$ and $\mathrm{AD}$, oxidative stresses may overwhelm antioxidant defense systems, thereby leading to neuronal cell death and dysfunction (29). ET or LA alone led to a significant decrease in the risks associated with AD and induced a significant improvement in antioxidant capabilities, which prevent subsequent neuronal cell loss $(16,21)$. The present study demonstrated that the SOD-1 and CAT protein levels were down-regulated in the brains of SED-Tg mice when compared with the Non-Tg mice, and that ET or LA alone led to up-regulation of the levels of these antioxidant enzymes over the SED-Tg control levels (Fig. 5).

These results suggest that ET has a better ability to induce antioxidant enzymes to cope with the superoxides formed in AD Tg mice. Furthermore, significantly higher SOD-1 and CAT protein levels were observed in mice subjected to ET alone than in SED-Tg mice, indicating that LA led to an effective reduction in oxidative insults. This finding is in accordance with several previous studies $(21,40,45)$. Notably, we recently $(13,16)$ demonstrated that combined application of ET and LA in APPsw mice was associated with greater improvements in antioxidant enzyme function in the brain than either treatment alone. Therefore, the results of the present study suggest that the reduced expression of SOD-1 and CAT is an important mechanism by which high reactive oxygen species stress occurs in the brains of $\mathrm{AD}$ patients, and that the combination of ET and LA can provide beneficial effects in the management of oxidative stress via up-regulation of SOD-1 and CAT.

HSP-70 has been reported to inhibit the self-assembly of polyglutamine proteins into amyloid like fibrils (46). However, it is not known whether an increase in HSP-70 within the brains of APPsw Tg mice occurs in response to ET and LA alone or in combination. Our study revealed that all of these treatments effectively restored the level of HSP-70 protein in the brains of Tg-mice, suggesting that ET or LA alone contributes to the neuroprotective effect in the brains of AD Tg-mice. Additionally, the increased levels of HSP-70 proteins following COMA may benefit the brain, making it more resistant to stress-induced cell damage. These results confirmed the results of several previously conducted studies $(47,48)$.

Finally, in this study, we found that APPsw Tg mice had higher total cholesterol, insulin and glucose levels in the serum than Non-Tg mice, which validated the results of previous studies $(13,49)$. Thus, the elevated circulating metabolic parameters may be directly connected to the elevation of Aß-42 levels, possibly via inhibition of glucose utilization, glucose transport or insulin signaling $(7,50)$. We also confirmed the results of other studies $(13,16,24)$ that found that reductions in metabolic parameters (TC, glucose and insulin) could be elicited by treating AD Tg-mice or diabetic rats with ET or LA alone. More importantly, the results of this study indicated that COMA resulted in a greater reduction in circulating glucose, insulin and cholesterol levels than either treatment alone. These results suggest that ET and LA ameliorates impaired glucose metabolism and insulin resistance while reducing cholesterol production in the brains of APPsw Tg mice. These effects likely occur via increased expression of the GLUT-1 protein and decreased levels of glucose, insulin and the deposition of $A B$ that may cause cognitive deficits.

In conclusion, ET and LA alone or in combination may represent a practical therapeutic management strategy for 
human subjects with AD. All of the aforementioned findings demonstrate that ET and LA alone or in combination can serve as a potential therapeutic method for preventing Aß-42 deposition and neuronal cell death and ameliorating the pathogenic phenotypes of AD. These results suggest that the combination with ET and LA showed a synergic effect on the improvement of behavioral defects, induction of BDNF and GLUT-1 expression, and the increase in antioxidant enzyme levels. However, in order to apply these methods in humans, further studies are needed to determine the benefits of ET and LA alone or in combination and the mechanisms underlying these beneficial effects.

\section{Acknowledgements}

We would like to thank the animal technicians H.-S. Bae, Y.-T. Shin, Y.-J. Hwang, Y.-H. Rho and J.-Y. Kim for their assistance at the animal facility of the Korea National Sport University. This study was supported by grants to Dr J.Y. Cho from the Korea Institute of Sport Science, Seoul Olympic Promotion Foundation (07-KISS-07-A06006).

\section{References}

1. Boland B and Campbell V: Beta-amyloid (1-40)-induced apoptosis of cultured cortical neurones involves calpainmediated cleavage of poly-ADP-ribose polymerase. Neurobiol Aging 24: 179-186, 2003.

2. McGrath LT, McGleenon BM, Brennan S, McColl D, McILroy S and Passmore AP: Increased oxidative stress in Alzheimer's disease as assessed with 4-hydroxynonenal but not malondialdehyde. QJM 94: 485-490, 2001

3. Monji A, Utsumi H, Ueda T, Imoto T, Yoshida I, Hashioka S, Tashiro K and Tashiro N: The relationship between the aggregational state of the amyloid-beta peptides and free radical generation by the peptides. J Neurochem 77: 1425-1432, 2001.

4. Gibson MS, Clarke EE, Zheng H, Van Der Ploeg LH, Ruffolo SC, Thornberry NA, Xanthoudakis S, Zamboni RJ, Roy S and Nicholson DW: Involvement of caspases in proteolytic cleavage of Alzheimer's amyloid-beta precusor protein and amyloidogenic A beta peptide formation. Cell 97: 395-406, 1999.

5. Fahnestock M, Garzon D, Holsinger RM and Michalski B: Neurotrophic factors and Alzheimer's disease: are we focusing on the wrong molecule? J Neural Transm 62: 241-252, 2002.

6. Lesne S, Gabriel C, Nelson DA, White E, Mackenzie ET, Vivien D and Buisson A: Akt-dependent expression of NAIP-1 protects neurons against amyloid-\{beta\} toxicity. J Biol Chem 280: 24941-24947, 2005.

7. Hooijmans CR, Graven C, Dederen PJ, Tanila H, van Groen T and Kiliaan AJ: Amyloid beta deposition is related to decreased glucose transporter-1 levels and hippocampal atrophy in brains of aged APP/PS1 mice. Brain Res 1181: 93-103, 2007.

8. Yoo BC, Kim SH, Cairns N, Fountoulakis M and Lubec G: Deranged expression of molecular chaperones in brains of patients with Alzheimer's disease. Biochem Biophys Res Commun 280: 249-258, 2001.

9. Cole SL, Grudzien A, Manhart IO, Kelly BL, Oakley H and Vassar R: Statins cause intracellular accumulation of amyloid precursor protein, beta-secretase-cleaved fragments, and amyloid beta-peptide via an isoprenoid-dependent mechanism. J Biol Chem 280: 18755-18770, 2005.

10. Watson GS and Craft S: Modulation of memory by insulin and glucose: neuropsychological observations in Alzheimer's disease. Eur J Pharmacol 490: 97-113, 2004.

11. Franklin CA and Karkeck J: Weight loss in Alzheimer's disease: an international review of the literature. Int Psychogeriatr 6: 135-142, 1989

12. Tamura BK, Masaki KH and Blanchette P: Weight loss in patients with Alzheimer's disease. J Nutr Elder 26: 21-38, 2007.
13. Cho JY, Hwang DY, Kang TS, Shin DH, Hwang JH, Lim CH, Lee SH, Lim HJ, Min SH, Seo SJ, Song YS, Nam KT, Lee KS, Cho JS, and Kim YK: Use of NSE/PS2m-transgenic mice in the study of the protective effect of exercise on Alzheimer's disease. J Sports Sci 21: 943-951, 2003.

14. Cotman CW, Berchtold NC and Christie LA: Exercise builds brain health: key roles of growth factor cascades and inflammation. Trends Neurosci 30: 464-472, 2007.

15. Colcombe SJ, Kramer AF, Erickson KI, Scalf P, McAuley E, Cohen NJ, Webb A, Jerome GJ, Marquez DX and Elavsky S: Cardiovascular fitness, cortical plasticity, and aging. Proc Natl Acad Sci USA 101: 3316-3321, 2004.

16. Um HS, Kang EB, Leem YH, Cho IH, Yang CH, Chae KR, Hwang DY and Cho JY: Exercise training acts as a therapeutic strategy for reduction of the pathogenic phenotypes for Alzheimer's disease in an NSE/APPsw-transgenic model. Int J Mol Med 22: 529-539, 2008

17. Packer L, Witt EH and Tritschler HJ: Alpha-lipoic acid as a biological antioxidant. Free Radic Biol Med 19: 227-250, 1995.

18. Konrad D, Somwar R, Sweeney G, Yaworsky K, Hayashi M, Ramlal T and Klip A: The antihyperglycemic drug alpha-lipoic acid stimulates glucose uptake via both GLUT4 translocation and GLUT4 activation: potential role of p38 mitogen-activated protein kinase in GLUT4 activation. Diabetes 50: 1464-1471, 2001.

19. Farr SA, Poon HF, Dogrukol-Ak D, Drake J and Banks WA, Eyerman E, Butterfield DA and Morley JE: The antioxidants alpha-lipoic acid and $\mathrm{N}$-acetylcysteine reverse memory impairment and brain oxidative stress in aged SAMP8 mice. J Neurochem 84: 1173-1183, 2003.

20. Liu J, Head E, Gharib AM, Yuan W, Ingersoll RT, Hagen TM, Cotman CW and Ames BN: Memory loss in old rats is associated with brain mitochondrial decay and RNA/DNA oxidation: partial reversal by feeding acetyl-L-carnitine and/or R-alpha-lipoic acid. Proc Natl Acad Sci USA 99: 2356-2361, 2002 .

21. Lovell MA, Xie C, Xiong S and Markesbery WR: Protection against amyloid beta peptide and iron/hydrogen peroxide toxicity by alpha lipoic acid. J Alzheimers Dis 5: 229-239, 2003.

22. Loske C, Neumann A, Cunningham AM, Nichol K, Schinzel R, Riederer P and Münch G: Cytotoxicity of advanced glycation endproducts is mediated by oxidative stress. J Neural Transm 105: 1005-1015, 1998.

23. Hwang DY, Cho JS, Lee SH, Chae KR, Lim HJ, Min SH, Seo SJ, Song YS, Song CW, Paik SG, Sheen YY and Kim YK: Aberrant expressions of pathogenic phenotype in Alzheimer's diseased transgenic mice carrying NSE-controlled APPsw. Exp Neurol 186: 20-32, 2004

24. Bitar MS, Wahid S, Pilcher CW, Al-Saleh and Al-Mulla F: Alpha-lipoic acid mitigates insulin resistance in Goto-Kakizaki rats. Horm Metab Res 36: 542-549, 2004.

25. Morris RG, Garrud P, Rawlins JN and O'Keefe J: Place navigation impaired in rats with hippocampal lesions. Nature 297: 681-683, 1982.

26. Qutub AA and Hunt CA: Glucose transport to the brain: a systems model. Brain Res Rev 49: 595-617, 2005.

27. Simpson IA, Chundu KR, Davies-Hill T, Honer WG and Davies, P: Decreased concentrations of GLUT1 and GLUT3 glucose transporters in the brains of patients with Alzheimer's disease. Ann Neurol 5: 511-521, 1994.

28. Gervais FG, Xu D, Robertson GS, Vaillancourt JP, Zhu Y, Huang J, LeBlanc A, Smith D, Rigby M, Shearman MS, Clarke EE, Zheng H, Van Der Ploeg LH, Ruffolo SC, Thornberry NA, Xanthoudakis S, Zamboni RJ, Roy S and Nicholson DW: Involvement of caspases in proteolytic cleavage of Alzheimer's amyloid-beta precursor protein and amyloidogenic A beta peptide formation. Cell 97: 395-406, 1999.

29. Noor R, Mittal S and Iqbal J: Superoxide dismutase applications and relevance to human diseases. Med Sci Monit 8: 210-215, 2002.

30. Warrick JM, Chan HY, Gray-Board GL, Chai Y, Paulson HL and Bonini NM: Suppression of polyglutamine-mediated neurodegeneration in Drosophila by the molecular chaperone HSP70. Nat Genet 23: 425-428, 1999.

31. Yenari MA, Liu J, Zheng Z, Vexler ZS, Lee JE and Giffard RG: Antiapoptotic and anti-inflammatory mechanisms of heat-shock protein protection. Ann NY Acad Sci 1053: 74-83, 2005.

32. Nelson TJ and Alkon DL: Insulin and cholesterol pathways in neuronal function, memory and neurodegeneration. Biochem Soc Trans 33: 1033-1036, 2005. 
33. Vance DE, Wadley VG, Ball KK, Roenker DL and Rizzo M: The effects of physical activity and sedentary behavior on cognitive health in older adults. J Aging Phys Act 13: 294-313, 2005.

34. Lytle ME, Vander BJ, Pandav RS, Dodge HH and Ganguli M: Exercise level and cognitive decline: the MoVIES project. Alzheimer Dis Assoc Disord 18: 57-64, 2004.

35. Cremer DR, Rabeler R, Roberts A and Lynch B: Long-term safety of alpha-lipoic acid (ALA) consumption: A 2-year study. Regul Toxicol Pharmacol 46: 193-201, 2006.

36. Lazarov O, Robinson J, Tang YP, Hairston IS, Korade-Mirnics Z, Lee VM, Hersh LB, Sapolsky RM, Mirnics K and Sisodia SS: Environmental enrichment reduces Abeta levels and amyloid deposition in transgenic mice. Cell 120: 7011-7020, 2005.

37. Quinn JF, Bussiere JR, Hammond RS, Montine TJ, Henson E, Jones RE and Stackman RW Jr: Chronic dietary alpha-lipoic acid reduces deficits in hippocampal memory of aged $\mathrm{Tg} 2576$ mice. Neurobiol Aging 28: 213-225, 2007.

38. Horwood N and Davies DC: Immunolabelling of hippocampal microvessel glucose transporter protein is reduced in Alzheimer's disease. Virchows Arch 425: 69-72, 1994.

39. Schultheiss C, Blechert B, Gaertner FC, Drecoll E, Mueller J, Weber GF, Drzezga A and Essler M: In vivo characterization of endothelial cell activation in a transgenic mouse model of Alzheimer's disease. Angiogenesis 9: 59-65, 2006.

40. Mattson MP and Magnus T: Ageing and neuronal vulnerability. Nat Rev Neurosci 7: 278-294, 2006.

41. Cechetti F, Rhod A, Simao F, Santin K, Salbego C, Netto CA and Siqueira IR: Effect of treadmill exercise on cell damage in rat hippocampal slices submitted to oxygen and glucose deprivation. Brain Res 1157: 121-125, 2007.

42. Cui X, Zuo P, Zhang Q, Li X, Hu Y, Long J, Packer L and Liu J: Chronic systemic D-galactose exposure induces memory loss, neurodegeneration, and oxidative damage in mice: protective effects of R-alpha-lipoic acid. J Neurosci Res 84: 647-654, 2006.
43. Craft SS, Asthana DG, Cook D, Baker M, Cherrier K, Purganan C, Wait A, Petrova S, Latendresse GS, Watson JW, Newcomer GD, Schellenberg and Krohn AJ: Insulin dose-response effects on memory and plasma amyloid precursor protein in Alzheimer's disease: interactions with apolipoprotein E genotype. Psychoneuroendocrinology 28: 809-822, 2003.

44. Nichol KE, Parachikova AI and Cotman CW: Three weeks of running wheel exposure improves cognitive performance in the aged Tg2576 mouse. Behav Brain Res 184: 124-132, 2007.

45. Jesudason EP, Masilamoni JG, Jebaraj CE, Paul SF and Jayakumar R: Efficacy of DL-alpha lipoic acid against systemic inflammation-induced mice: antioxidant defense system. Mol Cell Biochem 313: 113-123, 2008.

46. Muchowski PJ, Schaffar G, Sittler A, Wanker EE, Hayer-Hartl MK and Hartl FU: Hsp70 and hsp40 chaperones can inhibit selfassembly of polyglutamine proteins into amyloid-like fibrils. Proc Natl Acad Sci USA 97: 7841-7846, 2000.

47. Campisi J, Leem TH, Greenwood BN, Hansen MK, Moraska A, Higgins K, Smith TP and Fleshner M: Habitual physical activity facilitates stress-induced HSP72 induction in brain, peripheral, and immune tissues. Am J Physiol Regul Integr Comp Physiol 284: R520-R530, 2003.

48. Kinnunen S, Hyyppä S, Oksala N, Laaksonen DE, Hannila ML, Sen CK and Atala M: Alpha-lipoic acid supplementation enhances heat shock protein production and decreases post exercise lactic acid concentrations in exercised standard bred trotters. Free Radic Res 22: 1-9, 2009.

49. Sparks DL, Petanceska S, Sabbagh M, Connor D, Soares H, Adler C, Lopez J, Ziolkowski C, Lochhead J and Browne P: Cholesterol, copper and Abeta in controls, MCI, AD and the AD Cholesterol-Lowering Treatment Trial (ADCLT). Curr Alzheimer Res 2: 495-506, 2005.

50. De la Monte SM and Wands JR: Review of insulin and insulinlike growth factor expression, signaling, and malfunction in the central nervous system: relevance to Alzheimer's disease. J Alzheimer Dis 7: 45-61, 2005. 